\title{
Molecular pathways associated with transcriptional alterations in hyperparathyroidism
}

\author{
FANG LEE ${ }^{1}$, JIE-JEN LEE ${ }^{1-3}$, WOAN-CHING JAN ${ }^{2}$, CHIH-JEN WU ${ }^{3,4}$, \\ HAN-HSIANG $\mathrm{CHEN}^{3,4}$ and SHIH-PING CHENG ${ }^{1,3}$
}

\author{
${ }^{1}$ Department of Surgery, MacKay Memorial Hospital and MacKay Medical College, Taipei 10449; \\ ${ }^{2}$ Department of Nursing, MacKay Junior College of Medicine, Nursing and Management, Tapei 11260; \\ ${ }^{3}$ Department of Pharmacology and Graduate Institute of Medical Sciences, Taipei Medical University, Taipei 11031; \\ ${ }^{4}$ Department of Nephrology, MacKay Memorial Hospital and Mackay Medical College, Taipei 10449, Taiwan, R.O.C.
}

Received June 26, 2015; Accepted May 12, 2016

DOI: $10.3892 / \mathrm{ol} .2016 .4623$

\begin{abstract}
Hyperparathyroidism is characterized by the oversecretion of parathyroid hormone biochemically and increased cell proliferation histologically. Primary and secondary hyperparathyroidism exhibit distinct pathophysiology but share certain common microscopic features. The present study performed the first genome-wide expression analysis directly comparing the expression profile of primary and secondary hyperparathyroidism. Microarray gene expression analyses were performed in parathyroid tissues from 2 primary hyperparathyroidism patients and 3 secondary hyperparathyroidism patients. Unsupervised hierarchical clustering analysis identified two natural subgroups containing different types of hyperparathyroidism. Combined with additional data extracted from a publicly available database, a meta-signature was constructed to represent an intersection of two sets of differential expression profile. Multiple pathways were identified that are aberrantly regulated in hyperparathyroidism. In primary hyperparathyroidism, dysregulated pathways included cell adhesion molecules, peroxisome proliferator-activated receptor signaling pathway, and neuroactive ligand-receptor interaction. Pathways implicated in secondary hyperparathyroidism included tryptophan metabolism, tight junctions, renin-angiotensin system, steroid hormone biosynthesis, and O-glycan biosynthesis. The present study demonstrates that different pathophysiology is associated with differential gene profiling in hyperparathyroidism. Several pathways
\end{abstract}

Correspondence to: Dr Shih-Ping Cheng, Department of Surgery, MacKay Memorial Hospital and MacKay Medical College, 92 Chung-Shan North Road, Taipei 10449, Taiwan, R.O.C.

E-mail: surg.mmh@gmail.com

Key words: hyperparathyroidism, pathway analysis, cell adhesion, renin-angiotensin, metabolism are involved in parathyroid dysregulation and may be future targets for therapeutic intervention.

\section{Introduction}

Parathyroid hormone is the principal physiologic regulator of calcium homeostasis. Hyperparathyroidism is a result of increased activity of the parathyroid glands, either from an intrinsic change altering excretion of parathyroid hormone (primary hyperparathyroidism, pHPT) or from an extrinsic change affecting calcium homeostasis stimulating production of parathyroid hormone (secondary hyperparathyroidism, sHPT) (1). Clinically, hyperparathyroidism leads to skeletal and renal complications in addition to an impairment in quality of life (2-4). Prolonged oversecretion of parathyroid hormone is accompanied by histologically abnormal parathyroid glands that are typically enlarged and hypercellular with decreased stromal fat $(5,6)$.

Parathyroid glands are derived from the third and fourth pharyngeal pouches and are endodermal in origin (7). Generally, pHPT is caused by a single adenoma (80-85\%) or four-gland hyperplasia (10-15\%). Parathyroid carcinomas are rare and account for $<1 \%$ of pHPT cases. By contrast, four-gland hyperplasia is the rule in SHPT, ranging from diffuse chief cell hyperplasia to nodular formations. Significantly increased proliferation and apoptosis were both observed in pHPT and sHPT in comparison with normal parathyroids (8). Nonetheless, different genetic alterations are implicated in the development of different types of hyperparathyroidism $(9,10)$.

Although pHPT mainly occurs as a sporadic disease, it may be part of a hereditary syndrome (e.g., multiple endocrine neoplasia types 1 and $2 \mathrm{~A}$ ). On the other hand, chronic renal failure is the primary cause of SHPT. The phenomenon that different pathophysiology and genetic alterations of hyperparathyroidism lead to partially overlapping histological phenotype is notable. However, to the best of our knowledge, there have been no reports comparing transcriptional alterations in different types of hyperparathyroidism. In the present study, the gene expression differences between pHPT and sHPT were analyzed and molecular pathways that are dysregulated in different contexts of hyperparathyroidism were identified. 


\section{Materials and methods}

Patients and tissue samples. The present study was approved by the Institutional Review Board of MacKay Memorial Hospital (Taipei, Taiwan; approval no. 11MMHIS194), and all patients gave written informed consent. Parathyroid samples were obtained from patients undergoing surgical treatment of hyperparathyroidism at MacKay Memorial Hospital, Taipei, Taiwan (11). All samples were snap frozen in liquid nitrogen within 10 min of resection and stored at $-80^{\circ} \mathrm{C}$. Diagnosis was histologically confirmed by a senior endocrine pathologist using hematoxylin-eosin staining.

RNA extraction. Total RNA was extracted from homogenized frozen tissue samples using TRIzol reagent (Life Technologies, ThermoFisher Scientific, Inc., Waltham, MA, USA) and purified using the RNeasy Plus Mini Kit (Qiagen, Valencia, CA, USA) according to the manufacturer's recommendations (12). Sample purity was confirmed by measuring ratios of sample absorbance at 260 and $280 \mathrm{~nm}$ (ranging from 1.8 to 2.2). The quality of RNA was determined before labeling using the 2,100 Bioanalyzer (Agilent Technologies, Santa Clara, CA, USA).

Microarray hybridizations. A total of $200 \mu \mathrm{g}$ of total RNA was amplified by a Low Input Quick Amp Labeling Kit (Agilent Technologies) and labeled with $\mathrm{Cy} 3$ during the in vitro transcription process. Cy3-labled cRNA (600 $\mu \mathrm{g})$ was fragmented to an average size of $\sim 50-100$ nucleotides by incubation with fragmentation buffer at $60^{\circ} \mathrm{C}$ for $30 \mathrm{~min}$. Fragmented labeled cRNA was then pooled and hybridized to Agilent SurePrint G3 Human Gene Expression v2 8x60K Microarray at $65^{\circ} \mathrm{C}$ for $17 \mathrm{~h}$. After washing and drying, microarrays were scanned with an Agilent microarray scanner at $535 \mathrm{~nm}$ for Cy3. Scanned images were analyzed by Feature Extraction software version 10.5.1.1 (Agilent Technologies) to quantify signal and background intensity.

Data analysis and comparison with public microarray data. The microarray data were subjected to linear normalization to allow comparison between arrays. Hierarchical cluster analysis was performed with Cluster 3.0 (bonsai.hgc.jp/ mdehoon/software/cluster/software.htm), and heat maps were constructed with Java Treeview software (www.princeton.edu/ abarysh/treeview/). A public microarray dataset (GSE10317) was retrieved from the National Center for Biotechnology Information Gene Expression Omnibus (www.ncbi.nlm.nih.gov/geo/). GSE10317 comprises gene expression data of a case of pHPT (13). Gene expression levels of the parathyroid tumor and normal parathyroid tissue were analyzed using Affymetrix Human Genome U133 Plus 2.0 Arrays. (Affymetrix, Inc., Santa Clara, CA, USA)

$t$ statistics were used to estimate the significance of expression difference between pHPT and sHPT. R software version 3.0.2 (www.r-project.org) was used for Bayes-regularized $t$ tests. Associated P-values were adjusted for multiple testing by controlling for a false discovery rate $<5 \%$ using the Benjamini-Hochberg procedure (14), and adjusted $\mathrm{P}<0.05$ was considered to indicate a statistically significant difference. For the GSE10317 data, probes with a differential expression of at least 2 -fold were considered to be significant. A meta-signature that

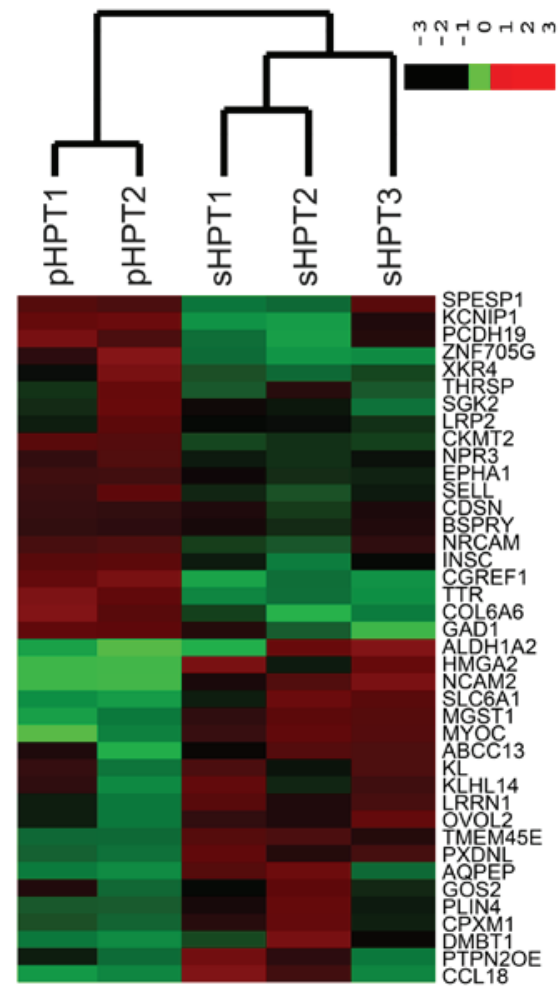

Figure 1. Hierarchical clustering of microarray data in patients with hyperparathyroidism.

characterized the intersection of differentially expressed genes from both datasets were constructed. Genes that demonstrated significantly altered expression changes in the same direction for both dataset were considered to be pHPT-associated. The intersection of differentially expressed genes of the two dataset in the opposite direction was considered to be sHPT-associated.

Gene Ontology (GO) terms and Kyoto Encyclopedia of Genes and Genomes (KEGG; www.genome.jp/kegg/) pathway analyses were performed to annotate the biological functions and pathways in which the aberrantly expressed genes of pHPT and sHPT were involved.

\section{Results}

Microarray gene expression analyses were performed in parathyroid tissues from 2 pHPT and 3 sHPT patients. The $2 \mathrm{pHPT}$ patients were female and had single parathyroid adenoma. All sHPT patients including 2 women and 1 man had four-gland nodular hyperplasia. Unsupervised hierarchical clustering analysis for the expression of all genes revealed two natural subgroups containing $\mathrm{pHPT}$ and sHPT, respectively.

A meta-signature was constructed to represent an intersection of two sets of differential expression profile. Based on predefined criteria, 339 genes were upregulated and 261 genes were downregulated in pHPT. The ten most common leading-edge genes are summarized in Tables I and II. A total of 218 genes were upregulated and 367 genes were downregulated in SHPT. The top upregulated and downregulated genes are shown in Tables III and IV, respectively. A heat map generated from the most differently expressed genes is presented in Fig. 1. 
Table I. Upregulated genes in primary hyperparathyroidism.

\begin{tabular}{|c|c|c|c|}
\hline Probe & Gene & Accession \# & Description \\
\hline A_23_P130333 & TTR & NM_000371 & Homo sapiens transthyretin \\
\hline A_33_P3814721 & INSC & NM_001031853 & $\begin{array}{l}\text { Homo sapiens inscuteable homolog (Drosophila), transcript } \\
\text { variant } 1\end{array}$ \\
\hline A_32_P224525 & COL6A6 & NM_001102608 & Homo sapiens collagen, type VI, alpha 6 \\
\hline A_33_P3400273 & SELL & NM_000655 & Homo sapiens selectin $\mathrm{L}$, transcript variant 1 \\
\hline A_24_P252364 & NRCAM & NM_001037132 & Homo sapiens neuronal cell adhesion molecule, transcript variant 1 \\
\hline A_23_P157333 & EPHA1 & NM_005232 & Homo sapiens $\mathrm{EPH}$ receptor A1 \\
\hline A_23_P350396 & CDSN & NM_001264 & Homo sapiens corneodesmosin \\
\hline A_33_P3244728 & LRP2 & NM_004525 & Homo sapiens low density lipoprotein receptor-related protein 2 \\
\hline A_23_P374689 & GAD1 & NM_000817 & $\begin{array}{l}\text { Homo sapiens glutamate decarboxylase } 1 \text { (brain, } 67 \mathrm{kDa} \text { ), } \\
\text { transcript variant GAD } 67\end{array}$ \\
\hline A_23_P71946 & BSPRY & NM_017688 & Homo sapiens B-box and SPRY domain containing \\
\hline
\end{tabular}

Table II. Downregulated genes in primary hyperparathyroidism.

\begin{tabular}{|c|c|c|c|}
\hline Probe & Gene & Accession \# & Description \\
\hline A_23_P36658 & MGST1 & NM_145791 & $\begin{array}{l}\text { Homo sapiens microsomal glutathione S-transferase 1, transcript } \\
\text { variant } 3\end{array}$ \\
\hline A_33_P3300253 & PTPN20B & NM_001042357 & $\begin{array}{l}\text { Homo sapiens protein tyrosine phosphatase, non-receptor type } \\
\text { 20B, transcript variant } 1\end{array}$ \\
\hline A_23_P74609 & G0S2 & NM_015714 & Homo sapiens $\mathrm{G} 0 / \mathrm{G} 1$ switch 2 \\
\hline A_33_P3251522 & AQPEP & NM_173800 & Homo sapiens laeverin \\
\hline A_33_P3400763 & PLIN4 & NM_001080400 & Homo sapiens perilipin 4 \\
\hline A_23_P23783 & MYOC & NM_000261 & $\begin{array}{l}\text { Homo sapiens myocilin, trabecular meshwork inducible } \\
\text { glucocorticoid response }\end{array}$ \\
\hline A_21_P0000096 & CPXM1 & NM_019609 & $\begin{array}{l}\text { Homo sapiens carboxypeptidase X (M14 family), member } 1 \text {, } \\
\text { transcript variant } 1\end{array}$ \\
\hline A_23_P258310 & PXDNL & NM_144651 & Homo sapiens peroxidasin homolog (Drosophila)-like \\
\hline A_23_P55270 & CCL18 & NM_002988 & $\begin{array}{l}\text { Homo sapiens chemokine (C-C motif) ligand } 18 \text { (pulmonary and } \\
\text { activation-regulated) }\end{array}$ \\
\hline A_23_P86599 & DMBT1 & NM_007329 & $\begin{array}{l}\text { Homo sapiens deleted in malignant brain tumors 1, transcript } \\
\text { variant } 2\end{array}$ \\
\hline
\end{tabular}

Table III. Upregulated genes in secondary hyperparathyroidism.

\begin{tabular}{|c|c|c|c|}
\hline Probe & Gene & Accession \# & Description \\
\hline A_24_P268685 & SLC6A1 & NM_003042 & $\begin{array}{l}\text { Homo sapiens solute carrier family } 6 \text { (neurotransmitter } \\
\text { transporter), member } 1\end{array}$ \\
\hline A_23_P503064 & KL & NM_004795 & Homo sapiens klotho \\
\hline A_24_P73577 & ALDH1A2 & NM_170697 & $\begin{array}{l}\text { Homo sapiens aldehyde dehydrogenase } 1 \text { family, member } \mathrm{A} 2 \text {, } \\
\text { transcript variant } 3\end{array}$ \\
\hline A_23_P1682 & TMEM45B & NM_138788 & Homo sapiens transmembrane protein $45 \mathrm{~B}$ \\
\hline A_23_P95930 & HMGA2 & NM_003483 & Homo sapiens high mobility group AT-hook 2 , transcript variant 1 \\
\hline A_24_P240187 & LRRN1 & NM_020873 & Homo sapiens leucine rich repeat neuronal 1 \\
\hline A_23_P143348 & OVOL2 & NM_021220 & Homo sapiens ovo-like zinc finger 2 \\
\hline A_32_P199429 & NCAM2 & NM_004540 & Homo sapiens neural cell adhesion molecule 2 \\
\hline A_23_P370830 & KLHL14 & NM_020805 & Homo sapiens kelch-like family member 14 \\
\hline A_23_P99253 & LIN7A & NM_004664 & Homo sapiens lin-7 homolog A (C. elegans) \\
\hline
\end{tabular}


Table IV. Downregulated genes in secondary hyperparathyroidism.

\begin{tabular}{|c|c|c|c|}
\hline Probe & Gene & Accession \# & Description \\
\hline A_23_P144778 & CKMT2 & NM_001825 & $\begin{array}{l}\text { Homo sapiens creatine kinase, mitochondrial } 2 \text { (sarcomeric), } \\
\text { transcript variant } 1\end{array}$ \\
\hline A_33_P3319248 & ZNF705G & NM_001164457 & Homo sapiens zinc finger protein $705 \mathrm{G}$ \\
\hline A_23_P131801 & SGK2 & NM_170693 & $\begin{array}{l}\text { Homo sapiens serum/glucocorticoid regulated kinase } 2 \text {, transcript } \\
\text { variant } 1\end{array}$ \\
\hline A_23_P30554 & KCNIP1 & NM_001034837 & Homo sapiens $\mathrm{Kv}$ channel interacting protein 1 , transcript variant 1 \\
\hline A_23_P129085 & SPESP1 & NM_145658 & Homo sapiens sperm equatorial segment protein 1 \\
\hline A_23_P363954 & THRSP & NM_003251 & Homo sapiens thyroid hormone responsive \\
\hline A_32_P134007 & XKR4 & NM_052898 & $\begin{array}{l}\text { Homo sapiens XK, Kell blood group complex subunit-related } \\
\text { family, member } 4\end{array}$ \\
\hline A_33_P3423230 & PCDH19 & NM_001184880 & Homo sapiens protocadherin 19, transcript variant 3 \\
\hline A_33_P3227793 & CGREF1 & NM_006569 & $\begin{array}{l}\text { Homo sapiens cell growth regulator with EF-hand domain 1, } \\
\text { transcript variant } 1\end{array}$ \\
\hline A_23_P58676 & NPR3 & NM_001204375 & $\begin{array}{l}\text { Homo sapiens natriuretic peptide receptor C/guanylate cyclase } \mathrm{C} \\
\text { (atrionatriuretic peptide receptor } \mathrm{C} \text { ), transcript variant } 1\end{array}$ \\
\hline
\end{tabular}

Table V. Pathway analysis of genes with differential expression in primary hyperparathyroidism.

\begin{tabular}{lccl}
\hline Pathway & Count & P-value & \multicolumn{1}{c}{ Genes } \\
\hline Cell adhesion molecules & 9 & 0.022 & $\begin{array}{l}\text { HLA-DQB1, NRCAM, ALCAM, SDC1, NRXN3, CD40LG, } \\
\text { SELL, ITGA8, ITGA4 }\end{array}$ \\
$\begin{array}{l}\text { PPAR signaling pathway } \\
\begin{array}{l}\text { Neuroactive ligand-receptor } \\
\text { interaction }\end{array}\end{array}$ & 6 & 0.034 & $\begin{array}{l}\text { HMGC, APOA5, APOC3, SLC27A6, ACSL3, SLC27A2 } \\
\text { CSH1, S1PR3, GABRG2, PTGER3, PRLR, RXFP1, HTR7, } \\
\text { GRIN2A, TAAR1, ADRA1A, GABBR2, PTGFR, GCGR }\end{array}$ \\
\hline
\end{tabular}

Table VI. Pathway analysis of genes with differential expression in secondary hyperparathyroidism.

\begin{tabular}{|c|c|c|c|}
\hline Pathway & Count & P-value & Genes \\
\hline Tryptophan metabolism & 6 & 0.003 & TDO2, CYP1B1, MAOA, AOX1, ALDH2, INMT \\
\hline Tight junction & 10 & 0.007 & $\begin{array}{l}\text { PRKCQ, INADL, MYH11, ACTN1, CLDN10, MYH7, } \\
\text { CLDN11, CTNNA3, CTNNA2, MYL9 }\end{array}$ \\
\hline Renin-angiotensin system & 4 & 0.009 & ACE2, MAS1, ANPEP, CTSG \\
\hline Steroid hormone biosynthesis & 5 & 0.030 & AKR1C3, CYP3A5, CYP1B1, AKR1C4, CYP19A1 \\
\hline O-glycan biosynthesis & 4 & 0.041 & GALNT3, GCNT1, GALNT13, GALNT14 \\
\hline
\end{tabular}

The gene function annotations were evaluated according to the GO and KEGG pathway databases. For genes with differential expression in pHPT, involved molecular functions in order were: Fatty-acid ligase activity, calcium ion binding, alkali metal ion binding, ligase activity forming carbon-sulfur bonds, and symporter activity. Involved biological processes were: Ion transport, cell adhesion, biological adhesion, cation transport, and metal ion transport. Dysregulated pathways in pHPT are presented in Table V.

Molecular interaction and networks contributing to SHPT were identified. Molecular functions involved in SHPT were: Retinal dehydrogenase activity, steroid dehydrogenase activity acting on the $\mathrm{CH}-\mathrm{OH}$ group of donors NAD or NADP as acceptor, carbohydrate binding, sulfotransferase activity, and acetylgalactosaminyltransferase activity. Involved biological processes were: Cell adhesion, biological adhesion, secondary metabolic process, skeletal system development, and regulation of nucleotide metabolic process. Dysregulated pathways in sHPT are listed in Table VI.

\section{Discussion}

The exact mechanism underlying the development of pHPT remains poorly understood (15). In patients with multiglandular pHPT, independent genetic events may be present in separate glands within the same individual (16). 
Previous studies have indicated that parathyroid adenomas typically harbor few somatic variants $(17,18)$. Mutations in the MEN1 tumor suppressor gene and alterations in the CCND1 (cyclin D1/PRAD1) oncogene represent the major driver in sporadic parathyroid tumorigenesis. Cell cycle regulators (including CDC73 aka HRPT2), growth factors, apoptosis-inducing ligands, death receptors, and other transmitter substances have also been implicated in the pathogenesis $(19,20)$. Although there is an abundance of data examining the gene expression profiles of parathyroid adenoma, few studies have been performed in comparing gene expressions of pHPT with sHPT tissues.

In a previous study examining clonality in $\mathrm{pHPT}$ and sHPT, 7/8 pHPT glands (6 adenomas and 2 hyperplasias) exhibited monoclonal proliferation (21). One parathyroid adenoma demonstrated a polyclonal pattern. In an sHPT patient, one of the 3 hyperplastic lesions was monoclonal and the other 2 lesions were polyclonal. This finding underscores the complexity of pHPT pathogenesis. The results from later studies also attest that $\mathrm{pHPT}$ can arise by clonal and polyclonal mechanisms (22). This is different from SHPT in which polyclonal growth transforms to monoclonal proliferation during disease progression (23). Nonetheless, there remains the possibility that similar mechanisms regulate parathyroid cell growth in both entities. The current study represents the first effort to compare the transcriptional profiles between pHPT and sHPT using modern microarray technology.

One of the limitations of the study design is the lack of transcriptional data from normal parathyroid controls. To overcome this ethical constraint, additional data were extracted from a publicly available database and combined to form a meta-signature. Regardless, the aberrantly regulated pathways that the present study identified in different types of hyperparathyroidism provide critical insights into the differences in pathophysiology. For instance, cell adhesion molecules were upregulated in pHPT but downregulated in SHPT. It is well known that nodular hyperplasia in patients with chronic kidney disease is associated with progressive downregulation of calcium-sensing receptor and vitamin D receptor (6). Activation of calcium-sensing receptor may potentiate cell adhesion by promoting integrin binding to a fibronectin-rich matrix (24). It is therefore reasonable that downregulated tight junction and cell adhesion were among the dysregulated pathways in SHPT. Conversely, expression of some adhesion molecules including selectin $\mathrm{L}$ and neuronal cell adhesion molecule were significantly increased in pHPT (Table I).

It was also noted that renin-angiotensin system was among the dysregulated pathways in sHPT. Increasing evidence links the renin-angiotensin-aldosterone system to calcium regulatory systems (25). A high calcium diet was demonstrated to downregulate angiotensin-converting enzyme of the kidney in experimental renal failure (26). In the present study, it was demonstrated that the expression of angiotensin I converting enzyme 2 (ACE2) was downregulated in SHPT. Recently, it has been shown that angiotensin II infusion acutely stimulated the secretion of parathyroid hormone in a dose-dependent manner (27). It is possible that downregulation of angiotensin-converting enzymes in sHPT results from a negative feedback mechanism to reduce further stimulation from angiotensin II. Renin-angiotensin system may be a potential target for therapeutic intervention in hyperparathyroidism.

The result of the present study point to alterations in peroxisome proliferator-activated receptor pathway in addition to fatty acid and amino acid metabolism in pHPT and sHPT. Metabolic aberrations have already been established as serving essential roles in imaging studies of hyperparathyroidism. Uptake and accumulation of technetium-99m sestamibi in mitochondria-rich oxyphil cells are employed as the basis of scintigraphic detection of hyperfunctional parathyroids (28). In addition, various tracers for positron emission tomographic scan have been exploited in localization of abnormal parathyroid glands (29,30). Metabolic reprogramming in neoplasms has recently been indicated as another general hallmark of cancer. Nonetheless, metabolic rearrangements in hyperparathyroidism remain a virtually untapped area of investigation. Elucidating the complex interplay between calcium homeostasis and parathyroid metabolic activity is an exciting challenge for future research.

In conclusion, the present study demonstrates that different pathophysiology led to differential gene profiling in hyperparathyroidism. Systemic analysis and annotated pathway resources were used to identify several pathways that are dysregulated in hyperparathyroidism which may be targets of interest.

\section{Acknowledgements}

The present study was supported by a grant (grant no. MOST103-2314-B-195-015-MY3) from the Ministry of Science and Technology of Taiwan. The funder had no role in study design, data collection and analysis, decision to publish, or preparation of the manuscript. Parts of this paper were presented at the 17th European Congress of Endocrinology (Dublin, Ireland, May 2015).

\section{References}

1. Fraser WD: Hyperparathyroidism. Lancet 374: 145-158, 2009.

2. Bilezikian JP, Brandi ML, Eastell R, Silverberg SJ, Udelsman R, Marcocci C and Potts JT Jr: Guidelines for the management of asymptomatic primary hyperparathyroidism: Summary statement from the fourth international workshop. J Clin Endocrinol Metab 99: 3561-3569, 2014.

3. Cheng SP, Lee JJ, Liu TP, Yang PS, Liu SC, Hsu YC and Liu CL: Quality of life after surgery or surveillance for asymptomatic primary hyperparathyroidism: A meta-analysis of randomized controlled trials. Medicine (Baltimore) 94: e931, 2015.

4. Cheng SP, Lee JJ, Liu TP, Yang TL, Chen HH, Wu CJ and Liu CL: Parathyroidectomy improves symptomatology and quality of life in patients with secondary hyperparathyroidism. Surgery 155 : 320-328, 2014

5. Elliott DD, Monroe DP and Perrier ND: Parathyroid histopathology: Is it of any value today? J Am Coll Surg 203: 758-765, 2006.

6. Drueke T, Martin D and Rodriguez M: Can calcimimetics inhibit parathyroid hyperplasia? Evidence from preclinical studies. Nephrol Dial Transplant 22: 1828-1839, 2007.

7. Bingham EL, Cheng SP, Woods Ignatoski KM and Doherty GM: Differentiation of human embryonic stem cells to a parathyroid-like phenotype. Stem Cells Dev 18: 1071-1080, 2009.

8. Thomopoulou GE, Tseleni-Balafouta S, Lazaris AC, Koutselini H, Kavantzas N and Davaris PS: Immunohistochemical detection of cell cycle regulators, Fhit protein and apoptotic cells in parathyroid lesions. Eur J Endocrinol 148: 81-87, 2003.

9. Shan L, Nakamura Y, Nakamura M, Yokoi T and Kakudo K: Genetic alterations in primary and secondary hyperparathyroidism. Pathol Int 48: 569-574, 1998. 
10. Westin G, Björklund P and Akerström G: Molecular genetics of parathyroid disease. World J Surg 33: 2224-2233, 2009.

11. Cheng SP, Lee JJ, Liu TP, Chen HH, Wu CJ and Liu CL: Aluminum overload hampers symptom improvement following parathyroidectomy for secondary hyperparathyroidism. World $\mathbf{J}$ Surg 38: 2838-2844, 2014.

12. Cheng SP, Yin PH, Chang YC, Lee CH, Huang SY and Chi CW: Differential roles of leptin in regulating cell migration in thyroid cancer cells. Oncol Rep 23: 1721-1727, 2010.

13. Au AY, McDonald K, Gill A, Sywak M, Diamond T, Conigrave AD and Clifton-Bligh RJ: PTH mutation with primary hyperparathyroidism and undetectable intact PTH. N Engl J Med 359: 1184-1186, 2008.

14. Benjamini Y and Yekutieli D: False discovery rate-adjusted multiple confidence intervals for selected parameters. J Am Stat Assoc 100: 71-81, 2005.

15. Cheng SP, Doherty GM, Chang YC and Liu CL: Leptin: The link between overweight and primary hyperparathyroidism? Med Hypotheses 76: 94-96, 2011.

16. Dwight T, Nelson AE, Theodosopoulos G, Richardson AL, Learoyd DL, Philips J, Delbridge L, Zedenius J, Teh BT, Larsson C, et al: Independent genetic events associated with the development of multiple parathyroid tumors in patients with primary hyperparathyroidism. Am J Pathol 161: 1299-1306, 2002.

17. Newey PJ, Nesbit MA, Rimmer AJ, Attar M, Head RT, Christie PT, Gorvin CM, Stechman M, Gregory L, Mihai R, et al: Whole-exome sequencing studies of nonhereditary (sporadic) parathyroid adenomas. J Clin Endocrinol Metab 97: E1995-E2005, 2012.

18. Costa-Guda J and Arnold A: Genetic and epigenetic changes in sporadic endocrine tumors: Parathyroid tumors. Mol Cell Endocrinol 386: 46-54, 2014

19. Lee JY, Kim SY, Mo EY, Kim ES, Han JH, Maeng LS, Lee AH, Eun JW, Nam SW and Moon SD: Upregulation of FGFR1 expression is associated with parathyroid carcinogenesis in HPT-JT syndrome due to an HRPT2 splicing mutation. Int J Oncol 45: 641-650, 2014

20. Segiet OA, Deska M, Michalski M, Gawrychowski J and Wojnicz R: Molecular profiling in primary hyperparathyroidism. Head Neck 37: 299-307, 2015.
21. Shan L, Nakamura M, Nakamura Y, Inoue D, Morimoto S, Yokoi T and Kakudo K: Comparative analysis of clonality and pathology in primary and secondary hyperparathyroidism. Virchows Arch 430: 247-251, 1997.

22. Shi Y, Hogue J, Dixit D, Koh J and Olson JA Jr: Functional and genetic studies of isolated cells from parathyroid tumors reveal the complex pathogenesis of parathyroid neoplasia. Proc Natl Acad Sci USA 111: 3092-3097, 2014.

23. Tominaga Y, Kohara S, Namii Y, Nagasaka T, Haba T, Uchida K, Numano M, Tanaka Y and Takagi H: Clonal analysis of nodular parathyroid hyperplasia in renal hyperparathyroidism. World J Surg 20: 744-750; discussion 750-752, 1996.

24. Tharmalingam S, Daulat AM, Antflick JE, Ahmed SM, Nemeth EF, Angers S, Conigrave AD and Hampson DR: Calcium-sensing receptor modulates cell adhesion and migration via integrins. J Biol Chem 286: 40922-40933, 2011.

25. Tomaschitz A, Ritz E, Pieske B, Rus-Machan J, Kienreich K, Verheyen N, Gaksch M, Grübler M, Fahrleitner-Pammer A, Mrak P, et al: Aldosterone and parathyroid hormone interactions as mediators of metabolic and cardiovascular disease. Metabolism 63: 20-31, 2014.

26. Porsti I, Fan M, Kööbi P, Jolma P, Kalliovalkama J, Vehmas TI, Helin H, Holthofer H, Mervaala E, Nyman T and Tikkanen I: High calcium diet down-regulates kidney angiotensin-converting enzyme in experimental renal failure. Kidney Int 66: 2155-2166, 2004.

27. Brown JM, Williams JS, Luther JM, Garg R, Garza AE, Pojoga LH, Ruan DT, Williams GH, Adler GK and Vaidya A: Human interventions to characterize novel relationships between the renin-angiotensin-aldosterone system and parathyroid hormone. Hypertension 63: 273-280, 2014.

28. Pons F, Torregrosa JV and Fuster D: Biological factors influencing parathyroid localization. Nucl Med Commun 24: 121-124, 2003.

29. Huang TS, Lee JJ, Lin YC and Cheng SP: Fluorodeoxyglucose-avid parathyroid adenoma mimicking thyroid incidentaloma. ANZ J Surg 80: 763-764, 2010.

30. Hindié E, Zanotti-Fregonara P, Tabarin A, Rubello D, Morelec I, Wagner T, Henry JF and Taïeb D: The role of radionuclide imaging in the surgical management of primary hyperparathyroidism. J Nucl Med 56: 737-744, 2015. 